\title{
A generalized Noether theorem for scaling symmetry
}

\author{
P.-M. Zhang ${ }^{1,2 *}$, M. Elbistan ${ }^{2,3 \dagger}$, P. A. Horvathy ${ }^{3 \ddagger}$ P. Kosiński ${ }^{4 \S}$ \\ 1 School of Physics and Astronomy, \\ Sun Yat-sen University Zhuhai 519082, China \\ 2 Institute of Modern Physics \\ Chinese Academy of Sciences, \\ Lanzhou, China \\ ${ }^{3}$ Institut Denis Poisson, \\ Tours University - Orléans University, \\ UMR 7013 (France). \\ 4 Department of Computer Science, \\ Faculty of Physics and Applied Informatics \\ University of Lódź, Pomorska 149/153, \\ 90-236 Lódź, Poland
}

(Dated: February 13, 2020)

* e-mail:zhangpm5@mail.sysu.edu.cn

$\dagger$ mailto:mahmut.Elbistan@lmpt.univ-tours.fr

$\ddagger$ mailto:horvathy@lmpt.univ-tours.fr

$\S$ email: pkosinsk@uni.lodz.pl 


\begin{abstract}
The recently discovered conserved quantity associated with Kepler rescaling is generalised by an extension of Noether's theorem which involves the classical action integral as an additional term. For a free particle the familiar Schroedinger-dilations are recovered. A general pattern arises for homogeneous potentials. The associated conserved quantity allows us to derive the virial theorem. The relation to the Bargmann framework is explained and illustrated by exact plane gravitational waves.
\end{abstract}

Eur. Phys. J. Plus (2020) 135:223

doi.org/10.1140/epjp/s13360-020-00247-5

PACS numbers:

11.30.-j Symmetry and conservation laws

11.10.Ef Lagrangian and Hamiltonian approach

45.50.Pk Celestial mechanics 


\section{Contents}

I. Introduction 3

II. A generalized Noether theorem 4

III. Hamiltonian framework 8

IV. Chrono-projective symmetries and gravitational waves 9

V. Gravitational waves and oscillators 11

$\begin{array}{ll}\text { VI. Discussion } & 14\end{array}$

$\begin{array}{ll}\text { Acknowledgments } & 15\end{array}$

$\begin{array}{ll}\text { References } & 16\end{array}$

\section{INTRODUCTION}

A Noether symmetry is a mapping $(\boldsymbol{q}, t) \rightarrow\left(\boldsymbol{q}^{\prime}, t^{\prime}\right)$ which leaves the Lagrangian $L$ invariant up to a surface term,

$$
L\left(\boldsymbol{q}^{\prime}, \frac{d \boldsymbol{q}^{\prime}}{d t^{\prime}}, t^{\prime}\right) \frac{d t^{\prime}}{d t} \rightarrow L\left(\boldsymbol{q}, \frac{d \boldsymbol{q}}{d t}, t\right)+\frac{d f(\boldsymbol{q}, t)}{d t}
$$

Then the consequences are twofold:

1. The classical Hamiltonian action $\int_{0}^{t} L d t$ changes by a path-independent term, implying that the variational equations remain invariant and thus a symmetry carries a motion into another motion.

2. Noether's theorem associates a conserved quantity to each continuous group of symmetries.

A classical "counter-example" when one, but not both consequences hold is provided by the rescaling of position and time in Kepler the problem :

$$
\begin{array}{lll}
t \rightarrow \lambda^{3} t, & \boldsymbol{q} \rightarrow \lambda^{2} \boldsymbol{q}, & \lambda=\text { const. } \\
\delta t \rightarrow 3(\delta \lambda) t, & \delta \boldsymbol{q} \rightarrow 2(\delta \lambda) \boldsymbol{q}, & \delta \lambda=\text { const. }
\end{array}
$$


which takes planetary trajectories into planetary trajectories, whereas the Lagrangian changes by a constant factor,

$$
L_{\text {Kepler }}=\frac{m}{2}\left(\frac{d \boldsymbol{q}}{d t}\right)^{2}+\frac{G m M_{\odot}}{|\boldsymbol{q}|} \rightarrow \lambda^{-2} L_{\text {Kepler }} .
$$

The rescaling (I.2) is therefore not a symmetry for the Keplerian system in the above sense and therefore no Noetherian conserved quantity is expected to arise ; textbooks call it a "similarity" [1]. It came therefore as a surprise that the Kepler problem does have a conserved quantity associated with (I.2) - which is however of a nonconventional form, involving also the classical Hamiltonian action [2],

$$
Q_{\text {Kepler }}=m \frac{d}{d t}\left(\boldsymbol{q}^{2}\right)-3 t E-S(t), \quad S(t)=\int_{0}^{t} L_{K e p l e r} d \tau,
$$

where the integration is along the classical trajectory in 3-space.

This novel conserved quantity which seems to have escaped attention until recently was obtained in [2] in a remarkably indirect way : the Kepler problem was first "E-D" (EisenhartDuval) lifted to a 5-dimensional "Bargmann" manifold [3-5]. See sec.IV for details.

The aim of this paper is to generalise this type of quantity by extending Noether's theorem first within the framework of analytical mechanics. Applications include, besides planetary motion, homogeneous potentials.

The relation to the Bargmann framework is explained in sec. IV. Particular attention is devoted to exact plane gravitational waves which correspond to time-dependent anisotropic oscillators $[6]$.

\section{A GENERALIZED NOETHER THEOREM}

Let us assume that we have a dynamical system with generalized coordinates $q_{i}, i=$ $1, \ldots, n$ and a Lagrangian $L=L(\boldsymbol{q}, \dot{\boldsymbol{q}}, t)$ and consider a 1-parameter family of transformations $\boldsymbol{q} \rightarrow \boldsymbol{q}^{\prime}\left(t^{\prime}\right), t \rightarrow t^{\prime}$, obeying

$$
L\left(\boldsymbol{q}^{\prime}, \frac{d \boldsymbol{q}^{\prime}}{d t^{\prime}}, t^{\prime}\right) \frac{d t^{\prime}}{d t}=\Lambda L\left(\boldsymbol{q}, \frac{d \boldsymbol{q}}{d t}, t\right)+\frac{d f(\boldsymbol{q}, t)}{d t}
$$

where $\Lambda$ is some constant and $f$ an arbitrary (differentiable) function. Then the associated Lagrange equations are invariant. Anticipating the terminology of Duval et al [7] discussed in sec. IV such transformations will be called Chrono-Projective. 
Then, proceeding in the standard way allows us to deduce from (II.1) the identity

$$
\frac{d}{d t}\left(\frac{\partial L}{\partial \dot{q}_{i}} \delta q_{i}-\left(\frac{\partial L}{\partial \dot{q}_{i}} \dot{q}_{i}-L\right) \delta t-\delta f\right)+\left(\frac{\partial L}{\partial q_{i}}-\frac{d}{d t}\left(\frac{\partial L}{\partial \dot{q}_{i}}\right)\right)\left(\delta q_{i}-\dot{q}_{i} \delta t\right)-(\delta \Lambda) L=0 .
$$

This relation is converted into a conservation law as follows. One solves the Lagrange equations of motion on some interval $0 \leq \tau \leq t$ with the "initial" [in fact final] conditions $q_{i}(t)=q_{i}$ and $\dot{q}_{i}(t)=\dot{q}_{i}$. Then the Hamiltonian action calculated along the trajectory,

$$
S=\int_{0}^{t} L(\boldsymbol{q}(\tau), \dot{\boldsymbol{q}}(\tau), \tau) d \tau
$$

becomes a function of the end points $q_{i}, \dot{q}_{i}$ and $t$; moreover, $d S / d t=L$. Inserting the Hamiltonian and using the Lagrange equations, eqn. (II.2) allows us deduce the conserved charge,

$$
Q=\frac{\partial L}{\partial \dot{q}_{i}} \delta q_{i}-H \delta t-\delta f-(\delta \Lambda) \int_{0}^{t} L d \tau
$$

Putting here (I.2b) and $\delta f=0$ yields, for example, the Kepler charge (I.4).

More generally, let's assume that (II.1) is satisfied (with $f=0$ for simplicity) and consider the Kepler-type rescaling

$$
\begin{aligned}
& q_{i}^{\prime}=\lambda^{a} q_{i} \approx q_{i}+a q_{i} \delta \lambda, \\
& t^{\prime}=\lambda^{b} t \approx t+b t \delta \lambda, \\
& \Lambda=\lambda^{c} \approx 1+c \delta \lambda .
\end{aligned}
$$

where $a, b, c$ are to be determined. ( $\approx$ means to first order).

Let us assume that the Lagrangian is of the form $L=g_{i j} \dot{q}_{i} \dot{q}_{j}-V(\boldsymbol{q})$, where for simplicity we took $g_{i j}$ a symmetric constant matrix. This Lagrangian(times $d t$ ) scales under (II.5a)(II.5b) as

$$
L d t \rightarrow \lambda^{2 a-b}\left[g_{i j} \dot{q}_{i} \dot{q}_{j}-\lambda^{-2 a+2 b} V\left(\lambda^{a} \boldsymbol{q}\right)\right] d t .
$$

Let us first turn off the potential, $V(\boldsymbol{q}) \equiv 0$, i.e., $L=L_{\text {free }}=\frac{1}{2} \dot{\boldsymbol{q}}^{2}$. Then (II.6) says that $L_{\text {free }} \rightarrow \lambda^{2 a-b} L_{\text {free }}$. The generalized symmetry condition (II.1) is thus satisfied with $\Lambda=\lambda^{c}$ i.e. if $c=2 a-b$. For any choice of $a$ and $b$ (II.4) associates a conserved charge, namely

$$
Q=a 2 g_{i j} \dot{q}^{i} q^{j}-b t H-c \int_{0}^{t} L d \tau, \quad c=2 a-b
$$


But $H_{\text {free }}=L_{\text {free }}$ and therefore $b$ drops out, and the last two terms combine into a single one :

$$
Q_{f r e e}=a\left(\dot{\boldsymbol{q}} \cdot \boldsymbol{q}-\dot{\boldsymbol{q}}^{2} t\right)=a \dot{\boldsymbol{q}} \cdot(\boldsymbol{q}-\dot{\boldsymbol{q}} t),
$$

where we recognise the scalar product of the (separately conserved) momentum with the center of mass. (II.8) quantity is actually the conserved quantity associated with Schrödinger dilations $[5,6,10,11], Q_{\text {free }}=D=\left(\dot{\boldsymbol{q}} \cdot \boldsymbol{q}-2 t H_{\text {free }}\right)$, traditionally obtained when time is dilated twice as much as space, i.e., $b=2 a$.

In the free case, this is the end of the story. Let us now restore the potential, $V$. The overall scaling $\Lambda$ is already determined by the kinetic term then (II.1) requires,

$$
\lambda^{-2(a-b)} V\left(\lambda^{a} \boldsymbol{q}\right)=V(\boldsymbol{q}) .
$$

For homogeneous potentials, $V(\mu \boldsymbol{q})=\mu^{k} V(\boldsymbol{q})$, the symmetry condition requires

$$
(-2+k) a+2 b=0 \quad \Rightarrow \quad c=a\left(1+\frac{k}{2}\right) .
$$

The integral term contributes whenever $k \neq-2$; the value of (II.7) is $Q=2 a g_{i j} q^{i}(0) \dot{q}^{j}(0)$.

When $a \neq 0 z=b / a$ is the dynamical exponent $[13,14])$; then $a=1$ can be chosen and the integral in (II.7) has coefficient $c=2-z$.

1. For free motion $k=0, a=b=c$, the terms combine, and we get (II.8).

2. For the inverse-square potential $V \propto|\boldsymbol{q}|^{-2}$ eqn. (II.10) requires $c=0$ i.e. $z=2$ and the Schrödinger scaling [10-12] is recovered.

3. For the Newtonian potential $V \propto|\boldsymbol{q}|^{-1}$ we have $k=-1$ and then get the Kepler scaling, $z=3 / 2$, cf. (I.2b) ; the integral term does not drop out from the Kepler charge (I.4) [2].

4. For the constant force $k=1$; the scaling is

$$
t \rightarrow \lambda t, \quad \boldsymbol{q} \rightarrow \lambda^{2} \boldsymbol{q}, \quad S \rightarrow \lambda^{3} S .
$$

It is amusing to figure that we climb, with Galilei, to the top of the Pisa Leaning Tower and drop a stone from $q(0)=0$. The conserved charge is

$$
Q_{\text {Gal }}=2 \boldsymbol{q}(t) \cdot \dot{\boldsymbol{q}}(t)-3 \int_{0}^{t} L_{\text {Gal }} d \tau, \quad L_{\text {Gal }}=\frac{1}{2} \dot{q}^{2}-q \Rightarrow Q_{\text {Gal }}=0 .
$$


5. For a harmonic oscillator $k=2$; (II.10) implies $b=0$, i.e., pure position-rescaling.

$$
Q_{o s c}=\boldsymbol{q}(t) \cdot \dot{\boldsymbol{q}}(t)-2 \int_{0}^{t} L_{o s c} d \tau, \quad L_{o s c}=\frac{1}{2} \dot{\boldsymbol{q}}^{2}-\frac{1}{2} \omega^{2} \boldsymbol{q}^{2} \Rightarrow Q_{o s c}=q_{i}(0) \dot{q}_{i}(0) .
$$

Let us study the oscillator case in some detail. After a full period $t=T=2 \pi / \omega$ we are back where we started from, so the first term in (II.13) vanishes. Therefore

$$
\frac{1}{T} \int_{0}^{T} \frac{1}{2} \dot{\boldsymbol{q}}^{2} d t=\frac{1}{T} \int_{0}^{T} \frac{1}{2} \omega^{2} \boldsymbol{q}^{2} d t
$$

i.e., the average over a period of the kinetic energy equals to the average of the potential energy - which is the virial theorem for an oscillator.

The virial theorem can actually be generalized to any $k$ along the same lines. For the standard Lagrangian/Hamiltonian $L=K-V$ resp. $H=K+V$ the associated conserved quantity (II.7) can be written as

$$
Q=a q_{i}(t) \dot{q}_{i}(t)-2 a \int_{0}^{t} K d \tau+2(a-b) \int_{0}^{t} V d \tau
$$

whose conservation implies for a periodic motion with period $T$

$$
<K>=\frac{1}{T} \int_{0}^{T} K d \tau=\frac{k}{2} \frac{1}{T} \int_{0}^{T} V d \tau=\frac{k}{2}<V>.
$$

We conclude this section by presenting an alternative point of view related to shape invariance. Let us assume that our Lagrangian $\widetilde{L}$ depends on an additional parameter $\mu$. Let a transformation $\boldsymbol{q}^{\prime}=\boldsymbol{q}^{\prime}(\boldsymbol{q}, t)$ and $t^{\prime}=t^{\prime}(\boldsymbol{q}, t)$ be completed by $\mu^{\prime}=\mu^{\prime}(\mu)$ and assume that we have, instead of (II.1),

$$
\widetilde{L}\left(\boldsymbol{q}^{\prime}, \frac{d \boldsymbol{q}^{\prime}}{d t^{\prime}}, t^{\prime}, \mu^{\prime}\right) \frac{d t^{\prime}}{d t}=\widetilde{L}\left(\boldsymbol{q}, \frac{d \boldsymbol{q}}{d t}, t, \mu\right)+\frac{d f(\boldsymbol{q}, t)}{d t} .
$$

Proceeding as before, we find the modified identity

$$
\frac{d}{d t}\left(\frac{\partial \widetilde{L}}{\partial \dot{q}_{i}} \delta q_{i}-\left(\frac{\partial \widetilde{L}}{\partial \dot{q}_{i}} \dot{q}_{i}-\widetilde{L}\right) \delta t-\delta f\right)+\left(\frac{\partial \widetilde{L}}{\partial q_{i}}-\frac{d}{d t}\left(\frac{\partial \widetilde{L}}{\partial \dot{q}_{i}}\right)\right)\left(\delta q_{i}-\dot{q}_{i} \delta t\right)+\frac{\partial \widetilde{L}}{\partial \mu} \delta \mu=0,
$$

which yields a "partial conservation law" for the usual conserved charge $\widetilde{Q}$,

$$
\widetilde{Q}=\frac{\partial \widetilde{L}}{\partial \dot{q}_{i}} \delta q_{i}-H \delta t-\delta f, \quad \frac{d \widetilde{Q}}{d t}+\frac{\partial \widetilde{L}}{\partial \mu} \delta \mu=0 .
$$

Choose in particular $\widetilde{L}=\mu L$, where $L$ is assumed to satisfy (II.1) with $\Lambda=1$ under the rescaling (II.5). The Lagrangians $L$ and $\widetilde{L}$ yield, for each fixed value of $\mu$, identical equations of motion. Our clue is that supplementing (II.5) with

$$
\mu^{\prime}=\lambda^{-c} \mu,
$$


the modified Lagrangian $\widetilde{L}$ becomes invariant. Moreover, $(\partial \widetilde{L} / \partial \mu) \delta \mu=c \mu \widetilde{L} \delta \lambda=\frac{d}{d t} \int^{t} \widetilde{L} d \tau$ and therefore, putting $\mu=1$, (II.19) allows us to recover the modified conserved quantity (II.4) as $Q=\widetilde{Q}-(\partial \widetilde{L} / \partial \mu)$.

\section{HAMILTONIAN FRAMEWORK}

In the Lagrangian framework, the Noether theorem applies to point transformations only. However it can also be reformulated in the Hamiltonian framework, leading to conserved charges generated by canonical symmetry transformations. The usual relation defining canonical transformations can be generalized in fact to include the scale factor $\Lambda$ as follows. The action integral becomes, after a Legendre transformation,

$$
\int\left[p_{i}^{\prime} \dot{q}_{i}^{\prime}-H^{\prime}\left(\boldsymbol{q}^{\prime}, \boldsymbol{p}^{\prime}, t\right)\right] d t=\int\left[\Lambda\left(p_{i} \dot{q}_{i}-H(\boldsymbol{q}, \boldsymbol{p}, t)\right)-\frac{d \Phi}{d t}\right] d t
$$

Putting $\Psi\left(\boldsymbol{q}, \boldsymbol{p}^{\prime}\right)=\Phi+q_{i}^{\prime} p_{i}^{\prime}$, this can be rewritten as

$$
\int\left[-q_{i}^{\prime} \dot{p}_{i}^{\prime}-H^{\prime}\right] d t=\int\left[\Lambda\left(p_{i} \dot{q}_{i}-H\right)-\frac{d \Psi}{d t}\right] d t
$$

which yields

$$
\begin{aligned}
& p_{i}=\frac{1}{\Lambda} \frac{\partial \Psi\left(\boldsymbol{q}, \boldsymbol{p}^{\prime}, t\right)}{\partial q_{i}}, \\
& q_{i}^{\prime}=\frac{\partial \Psi\left(\boldsymbol{q}, \boldsymbol{p}^{\prime}, t\right)}{\partial p_{i}^{\prime}}, \\
& H^{\prime}=\Lambda H+\frac{\partial \Psi\left(\boldsymbol{q}, \boldsymbol{p}^{\prime}, t\right)}{\partial t}
\end{aligned}
$$

The identity transformation is generated by the function $\Psi_{0}=q_{i} p_{i}^{\prime}$ and $\Lambda=1$. Therefore the infinitesimal transformation is obtained by putting $\Lambda=1+\delta \Lambda$ and

$$
\Psi\left(\boldsymbol{q}, \boldsymbol{p}^{\prime}, t\right)=\Psi_{0}\left(\boldsymbol{q}, \boldsymbol{p}^{\prime}\right)+\delta G\left(\boldsymbol{q}, \boldsymbol{p}^{\prime}, t\right)=\Psi_{0}\left(\boldsymbol{q}, \boldsymbol{p}^{\prime}\right)+\delta G(\boldsymbol{q}, \boldsymbol{p}, t)
$$

where in we replaced $\boldsymbol{p}^{\prime}$ by $\boldsymbol{p}$ because $\delta G$ is already infinitesimal. Then our eqns yield

$$
\begin{aligned}
& \delta p_{i}=p_{i}^{\prime}-p_{i}=\delta \Lambda p_{i}-\frac{\partial(\delta G)}{\partial q_{i}} \\
& \delta q_{i}=q_{i}^{\prime}-q_{i}=\frac{\partial(\delta G)}{\partial p_{i}} \\
& H^{\prime}=H+\delta \Lambda H+\frac{\partial(\delta G)}{\partial t}
\end{aligned}
$$


A canonical transformation is a symmetry if the Hamiltonian equations are form-invariant, $H^{\prime}\left(\boldsymbol{q}^{\prime}, \boldsymbol{p}^{\prime}, t\right)=H\left(\boldsymbol{q}^{\prime}, \boldsymbol{p}^{\prime}, t\right)$. Expanding to the first order and using (III.5) one finds,

$$
\{\delta G, H\}+\frac{\partial(\delta G)}{\partial t}+\delta \Lambda\left(H-p_{i} \frac{\partial H}{\partial p_{i}}\right)=0 \text { i.e., } \quad \frac{d}{d t}\left(\delta G-\delta \Lambda \int_{0}^{t} L d \tau\right)=0
$$

which is the generalized Noether charge.

For a point transformation we get, for $\delta f=0$,

$$
\delta G=\frac{\partial L}{\partial \dot{q}_{i}} \delta q_{i}-H \delta t=p_{i} \delta q_{i}-H \delta t .
$$

In the Lagrangian framework time and space transformations can be combined. However time is fixed in the Hamiltonian one, and so in order to include time-variable transformations, we have to replace $\delta q_{i}, \delta p_{i}$ by

$$
\begin{aligned}
& \delta_{H} q_{k}=\left\{q_{k}, \delta G\right\}=\delta q_{k}-\dot{q}_{k} \delta t \\
& \delta_{H} p_{k}=\left\{p_{k}, \delta G\right\}=-p_{i} \frac{\partial\left(\delta q_{i}\right)}{\partial q_{k}}-\dot{p}_{k} \delta t
\end{aligned}
$$

i.e., for point transformations, $q_{k}$ and $p_{k}$ are corrected by a time shift. But in the Hamiltonian framework Noether's theorem is more general : $\delta G$ can have a more complicated form, and generate canonical transformations which can not be derived from point transformations.

We note that for the scaling transformations (II.5) the generator $\delta G$ in (III.4)-(III.5) could be expressed in terms of the initial conditions as $\delta G=a q_{i}(0) \dot{q}_{i}(0) \delta \lambda$.

\section{CHRONO-PROJECTIVE SYMMETRIES AND GRAVITATIONAL WAVES}

A convenient way to study non-relativistic conformal symmetries is to use the "Bargmann" framework [4, 5] : one lifts the non-relativistic dynamics in $(d, 1)$ dimensions to a $(d+2)$ dimensional manifold $M$ endowed with a Lorentz metric $g_{\mu \nu}$ and a covariantly constant null vector $\xi$, referred to as a Bargmann space [4, 5]. The original non-relativistic motions are projections of null geodesics lying "upstairs", i.e., in Bargmann space. For motion in a potential $V(q, t)$ the appropriate metric resp. "vertical vector" are

$$
g_{\mu \nu} d x^{\mu} d x^{\nu}=d \boldsymbol{q}^{2}+2 d t d s-2 V d t^{2} \quad \text { and } \quad \xi=\partial_{s}
$$


The vertical vector $\xi$ generates and isometry, whose conserved momentum associated with the Killing vector $\partial_{s}$ upstairs, $P_{s}$ is the physical mass "downstairs" i.e., in $2 D$ non-relativistic spacetime.

The remarkable fact recognized already by Eisenhart [3] is that the null lift of a nonrelativistic motion (we call the Eisenhart-Duval (E-D) lift) is,

$$
\left(q^{i}(t), t, s_{0}-S(t)\right) \quad \text { where } \quad S(t)=\int_{0}^{t} L_{c l}(q(\tau), \tau,) d \tau
$$

i.e., the "vertical" coordinate, $s$ is essentially i.e. up to a constant minus the classical Hamiltonian action calculated along the trajectory. It is precisely this rule (IV.2) which guarantees that the lift is null, as shown by evaluating (IV.1) for a tangent vector.

Conformal transformations of $\left(M, g_{\mu \nu}\right)$ take null-geodesics into null-geodesics; however such a transformation generated by a vector field $Y$ projects to a symmetry for the nonrelativistic dynamics "downstairs" only if it satisfies the additional condition

$$
L_{Y} \xi=0
$$

For the Minkowski metric written in light-cone coordinates, for example, (IV.1) with $V=0$, the conformal algebra is $\mathrm{o}(d+1,2)$ and those vectorfields which satisfy (IV.3) span the centrally extended Schrödinger group [4,5]. Schrödinger dilations are, in particular, identical to $Q_{\text {free }}$ in (II.8).

The Kepler rescaling (I.2) can be lifted to Bargmann space [with $V=-G M_{\odot} /|\boldsymbol{q}|$ ], as

$$
t \rightarrow \lambda^{3} t, \boldsymbol{q} \rightarrow \lambda^{2}, s \rightarrow \lambda s \quad \text { infinitesimally } \quad Y=3 t \partial_{t}+2 \boldsymbol{q} \cdot \partial_{\boldsymbol{q}}+s \partial_{s},
$$

becoming there a conformal transformation; the scaling of the coordinate $s$ is dictated precisely by this requirement $[2,6,9]$.

However (IV.4) does not satisfy (IV.3) $: L_{Y} \xi=-\delta \lambda \xi$. Transformations of the Bargmann space such that $L_{Y} \xi$ is parallel to $\xi$,

$$
L_{Y} \xi=\psi \xi
$$

for some (necessarily constant) $\psi$ are in fact the lifts of chrono-projective transformations, originally introduced in terms of the Newton-Cartan structure "downstairs [7, 8]. These same condition was also considered, independently, by Hall et al [15] in their study of conformal transformations for gravitational waves. 
Conformal transformations are symmetries for null geodesics upstairs and generate there, through Noether's theorem, conserved quantities - but the associated conserved charge may not project to ordinary space-time as well-defined quantities. However, when the additional "Chrono-projective" condition (IV.5) is satisfied, then they fail to project by just a little: it is enough to subtract a constant term proportional to the initial value $s_{0}$ to get a perfectly well-defined conserved quantity for the projected dynamics [2, 6]. This is what happens for (I.2) : eqn. (IV.2) implies that

$$
Q_{\text {Kepler }}=\mathcal{Q}_{\text {Kepler }}-m s_{0} \quad \text { where } \quad \mathcal{Q}_{\text {Kepler }}=-3 t E+2 m \boldsymbol{q} \cdot \dot{\boldsymbol{q}}+m s
$$

consistently with (I.4).

1-dim oscillator. An even simpler illustration is provided by a 1-d harmonic oscillator. Its $3 \mathrm{~d}$ Bargmann space has the metric is $d s^{2}=d q^{2}-2 d t d s-\omega^{2} q^{2} d t^{2}$ and $\xi=\partial_{s}$. The space-alone rescaling lifts to $3 d$ Bargmann space as the homothety,

$$
t \rightarrow t, \quad q \rightarrow \lambda q, \quad s \rightarrow \lambda^{2} s
$$

and generates there a chrono-projective symmetry for null geodesics $[2,5,6,8]$, as shown on Fig.1. The associated conserved quantity is (II.13).

Let us consider two motions $q_{1}(t)$ and $q_{2}(t)$ which start from the same initial position $q_{1}(0)=0=q_{2}(0)$ but with different initial velocities, $\dot{q}_{2}(0)=\lambda \dot{q}_{1}(0)$. The space-alone rescaling $q \rightarrow \lambda q, t \rightarrow t$ takes $q_{1}(t)$ into $q_{2}(t)$. Both motions return to their initial position after a half period (since the latter is independent of the initial conditions, as purportedly observed by Galilei in the Pisa cathedral).

\section{GRAVITATIONAL WAVES AND OSCILLATORS}

Let us now consider the exact gravitational plane wave studied by Brinkmann [16],

$$
\begin{array}{r}
g_{\mu \nu} d x^{\mu} d x^{\nu}=d \boldsymbol{q}^{2}+2 d t d s+K_{i j}(t) q^{i} q^{j} d t^{2} \\
K_{i j}(t) q^{i} q^{j}=\frac{1}{2} \mathcal{A}_{+}(t)\left(\left(q^{1}\right)^{2}-\left(q^{2}\right)^{2}\right)+\mathcal{A}_{\times}(t) q^{1} q^{2}
\end{array}
$$

where $\mathcal{A}_{+}$and $\mathcal{A}_{\times}$are the + and $\times$polarization-state amplitudes. A simple example is given by the $t$-independent linearly polarized gravitational wave [17] with $\mathcal{A}_{+}=2 \Omega^{2}, \Omega=$ const. 


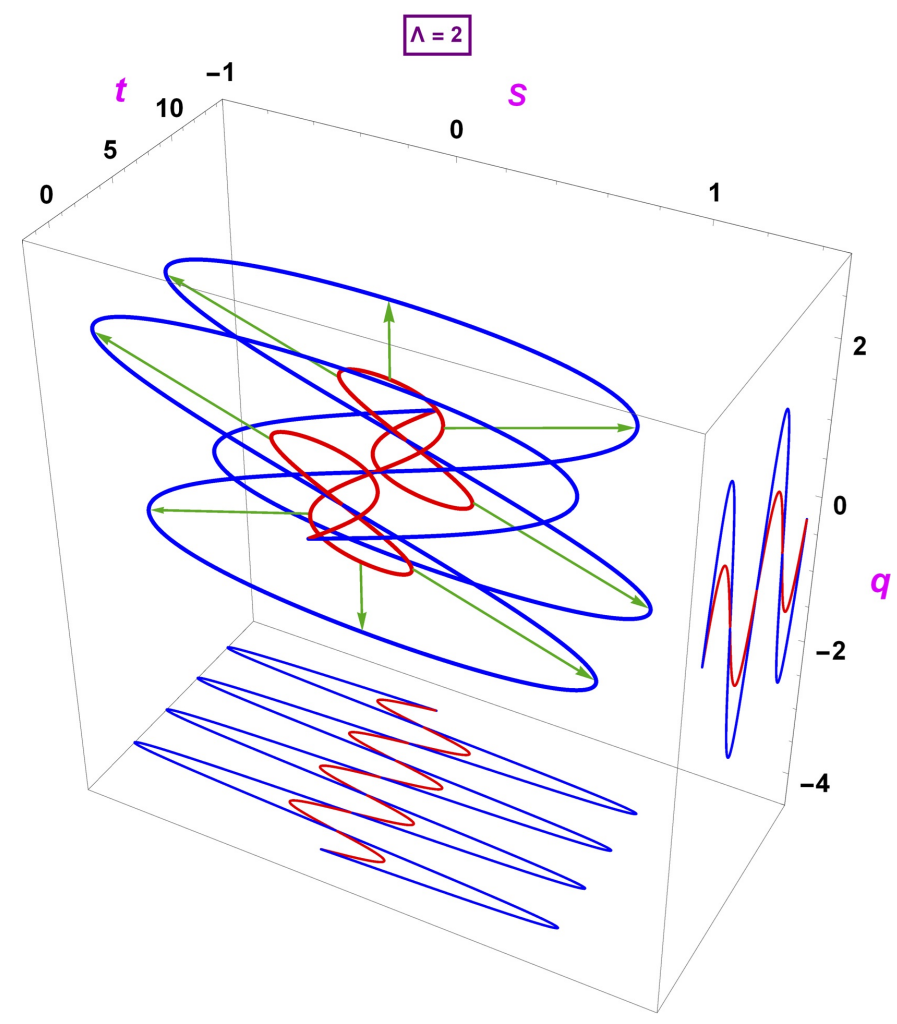

FIG. 1: The Eisenhart-Duval lifts to 3d Bargmann space of two motions of a 1d harmonic oscillator. Projected to the $(q, t)$ plane [which is here vertical] we get the familiar sinus curves related by position-alone scaling by $\lambda$. The Hamiltonian actions calculated along the trajectories, shown in the $(S, t)$ [here horizontal] plane oscillate with double frequency and are scaled by $\lambda^{2}$. The E-D lift of the scaling [in green], (IV.7), the homothety carries the lifted curves into each other.

and $\mathcal{A}_{\times}=0$, i.e.,

$$
g_{\mu \nu} d x^{\mu} d x^{\nu}=d \boldsymbol{q}^{2}+2 d t d s-\Omega^{2}\left(\left(q^{1}\right)^{2}-\left(q^{2}\right)^{2}\right) d t^{2}
$$

Viewed as a Bargmann space, this metric describes an attractive oscillator in the $q^{1}$ coordinate combined with a repulsive (inverted) one in the $q^{2}$ sector ; $t$ corresponds to nonrelativistic time (as anticipated). The motion is governed by the geodesic Lagrangian

$$
\frac{1}{2}\left(\left(\dot{q}^{1}\right)^{2}+\left(\dot{q}^{2}\right)^{2}\right)+\dot{t} \dot{V}-\frac{1}{2} \Omega^{2}\left(\left(q^{1}\right)^{2}-\left(q^{2}\right)^{2}\right)
$$

where the dot denotes derivation w.r.t. an affine parameter. For a particle initially at rest (e.g. with initial conditions $\left.q^{1}(0)=q^{2}(0)=1 \dot{q}^{1}(0)=\dot{q}^{2}(0)=0\right)$ the trajectory is,

$$
\left\{\begin{array}{l}
q^{1}(t)=\cos \Omega t \\
q^{2}(t)=\cosh \Omega t
\end{array} \quad s(t)=s_{0}+\frac{\Omega}{4}(\sin 2 \Omega t-\sinh 2 \Omega t),\right.
$$


shown on fig.2. Then the homothety (IV.7) $[6,18,19]$ generated by

$$
Y_{h o m}=q^{i} \partial_{i}+2 s \partial_{s}
$$

is a chrono-projective transformation [8] of the metric (V.2),

$$
L_{Y_{\text {hom }}} g=2 \chi g, \quad L_{Y_{\text {hom }}} \xi=-2 \chi \xi
$$

The associated conserved charge is

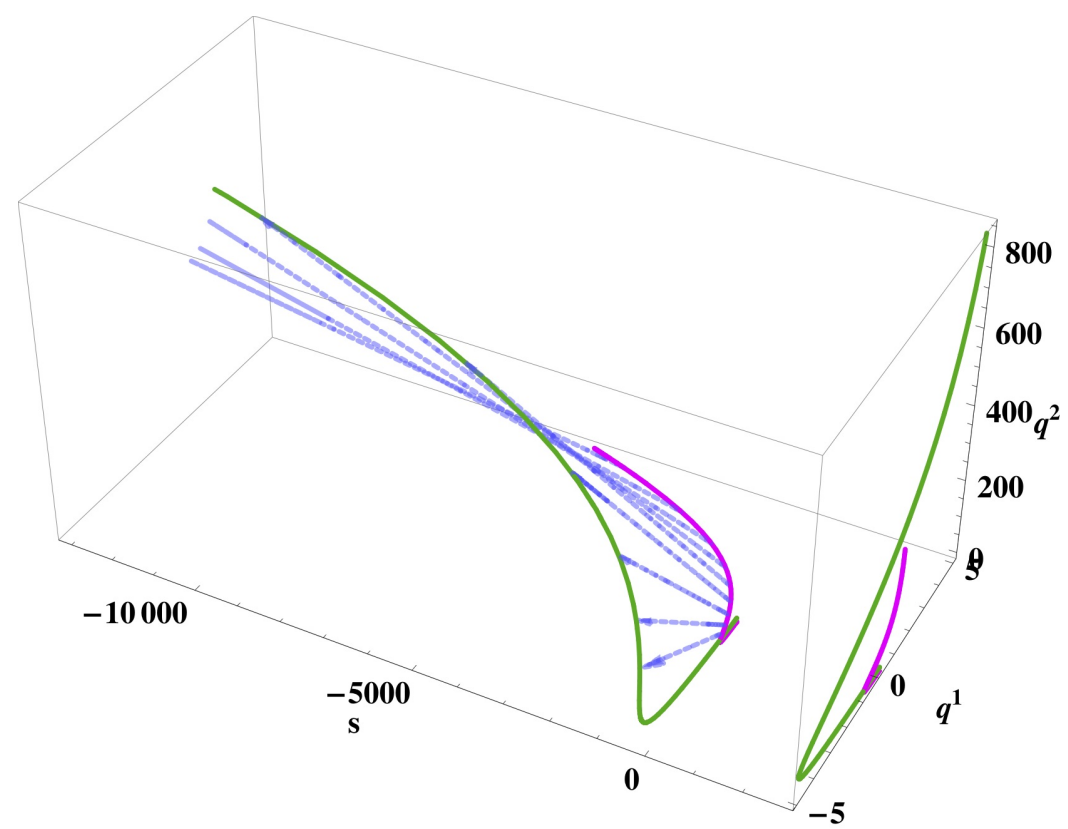

FIG. 2: The Brdička metric (V.2) provides us with the Bargmann description of a particle moving in a saddle potential. The trajectory combines oscillation in the attractive $q^{1}$ sector with exponentially escaping motion in the repulsive $q^{2}$ and s sectors. The trajectories are carried into each other by the homothety (V.5).

$$
\begin{aligned}
& Q_{\text {hom }}=\mathcal{Q}-2 s_{0}=\boldsymbol{q}(t) \cdot \dot{\boldsymbol{q}}(t)-2 S(t) \\
& S(t)=\int_{0}^{t} L_{o s c}(\boldsymbol{q}(\tau), \dot{\boldsymbol{q}}(\tau)) d \tau, \quad L_{o s c}=\frac{1}{2} \dot{\boldsymbol{q}}^{2}-\frac{1}{2} \Omega^{2}\left(\left(q^{1}\right)^{1}-\left(q^{2}\right)^{2}\right) .
\end{aligned}
$$

Evaluating the integral, $Q_{\text {hom }}=(\boldsymbol{q} \cdot \dot{\boldsymbol{q}})(0)=0$.

We remark that changing the relative sign from minus to plus in (V.2),

$$
K_{i j}(t) q^{i} q^{j}=\frac{1}{2} \Omega^{2}\left(\left(q^{1}\right)^{2}+\left(q^{2}\right)^{2}\right), \quad \Omega=\text { const. }
$$


would yield instead the Bargmann description of a time independent isotropic harmonic oscillator ${ }^{1}$. The familiar elliptic trajectories in the transverse plane lift to $4 D$ Bargmann space as null geodesics ; the $\xi$-preserving isometries span the centrally extended Newton-Hooke group, whose $\xi$-preserving conformal extension spans a group isomorphic to the Schrödinger group, etc. Here we just mention that the homothety (V.5) acts as a chrono-projective symmetry. The associated Noether charge is still $\mathcal{Q}_{h o m}=q^{i} P_{i}+2 s P_{s}$, yielding the projected conserved quantity (V.7a)

$$
Q_{o s c}=q^{i} P_{i}-2 \int_{0}^{t} L_{o s c} d \tau=\boldsymbol{q}(0) \cdot \dot{\boldsymbol{q}}(0)
$$

with the isotropic oscillator Lagrangian $L_{\text {osc }}=\frac{1}{2} \dot{\boldsymbol{q}}^{2}-\frac{1}{2} \Omega^{2} \boldsymbol{q}^{2}$. Fig.3 should be compared with the Kepler figure in [2].

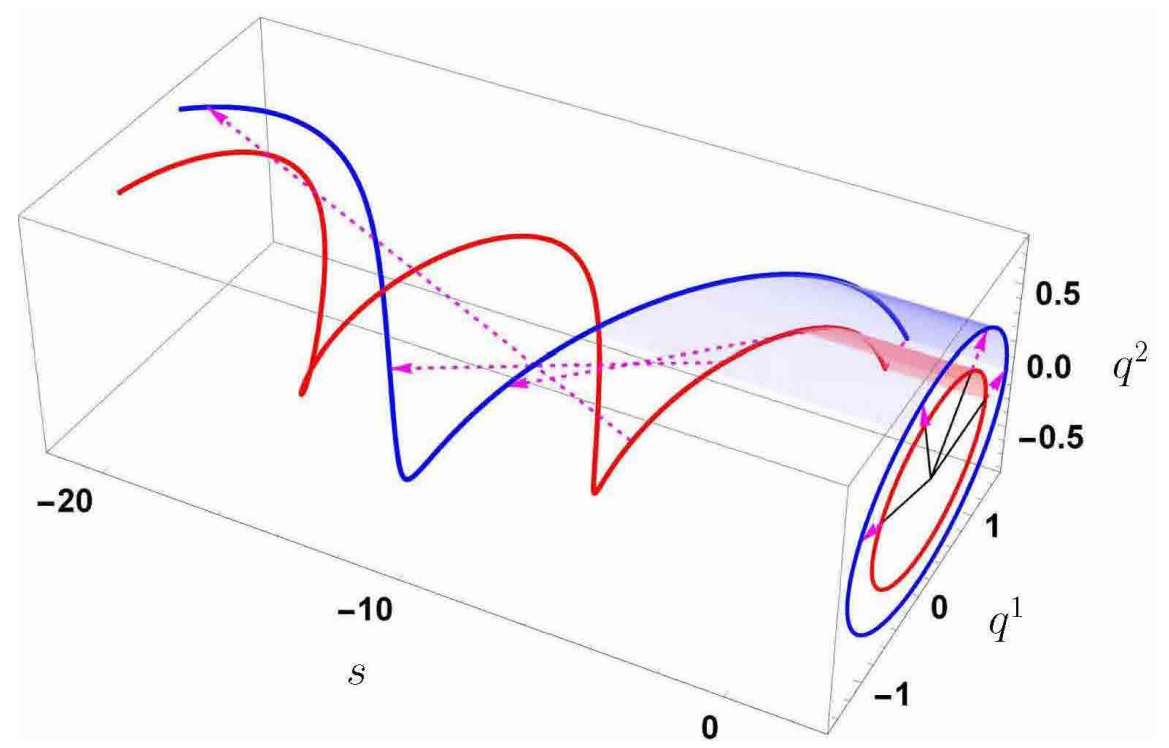

FIG. 3: Motion of a $2 d$ oscillator unfolded to $4 D$ Bargmann space [and dropping the non-relativistic time coordinate]. The homothety (V.5) carries lifted oscillator-ellipses to lifted oscillator-ellipses.

\section{DISCUSSION}

In this paper we extended Noether's theorem to more general symmetry transformations which include also rescalings. Our results confirm the conserved charge found recently [2] for the Kepler problem; for a free particle we recover Schrödinger dilations [10, 11]. For

1 (V.8) is only a pp wave, not a vacuum solution of the Einstein equations $[5,16]$. 
homogeneous potential (which include free fall and a harmonic oscillator) we get a new charge, whose conservation allows us we rederive the virial theorem. Further applications (namely to gravitational plane waves) will be presented elsewhere [6].

Compared with the usual Noether theorem, our new charge (II.4) has has an extra term, in fact the classical action, which can be calculated only after the equations of motion had been solved. The new conservation law has nevertheless useful applications, as demonstrated by its use to prove the virial theorem and to derive Kepler's Third law [2].

Further applications and generalizations are discussed in [6] where it is argued that a similar conserved quantity arises for exact plane waves, and behaves as a symmetry for null geodesics motion [20-22]. The extension to massive geodesics is considered in [23].

We just mention that Maxwell's electromagnetic Lagrangian under duality transformations would provide a field-theoretical example with helicity as associated conserved quantity.

After our paper was first posted to arXiv, our attention was called to earlier investigations $[24,25]$. The closest to our approach is that van Kampen [24], who uses also a Lagrangian framework; his equation \# (5) is in fact our eqn. (II.1). He could have [but did not] obtain our new charge (II.4) by integrating his unnumbered equation after his \# (5) on p.237.

Nachtergaele et al. [25] studied canonical transformations in the Hamiltonian framework in symplectic space, and applied them to Toda chains.

Both of these papers focus on the virial theorem related to the Kepler rescaling ; no additional conserved quantity was found, though.

We also came across [26] which uses Lie transformations and would also allow to derive our new charge (II.4). Our approach here is based instead on chrono-projective transformations $[7,8]$ in the context of E-D lifts to Bargmann manifolds, which are gravitational wave spacetimes [3-5]. Also ref. [27] discusses similar issues.

\section{Acknowledgments}

We are indebted to Gary Gibbons for advice and Bruno Nachtergaele for correspondance. ME thanks the Denis Poisson Institute of Orléans - Tours University for a post-doctoral scholarship. This work was partially supported by National Natural Science Foundation of China (Grant No. 11575254). P.K. was supported by the grant 2016/23/B/ST2/00727 of the National Science Centre of Poland. 
[1] L. Landau and E. Lifchitz, Physique Théorique, Tome I: Mécanique. $3^{e}$ édition. Éditions MIR, Moscou (1969); V. Arnold, Les méthodes mathématiques de la mécanique classique, Éditions MIR, Moscou (1976).

[2] P.-M. Zhang, M. Cariglia, M. Elbistan, G. W. Gibbons and P. A. Horvathy, "'Kepler Harmonies" and conformal symmetries," Physics Letters B 792 (2019) 324 [arXiv:1903.01436 [gr-qc]]. https://doi.org/10.1016/j.physletb.2019.03.057

[3] L. P. Eisenhart, "Dynamical trajectories and geodesics", Annals Math. 30 591-606 (1928).

[4] C. Duval, G. Burdet, H. P. Künzle and M. Perrin, "Bargmann structures and Newton-Cartan theory", Phys. Rev. D 31 (1985) 1841.

[5] C. Duval, G.W. Gibbons, P. Horvathy, "Celestial mechanics, conformal structures and gravitational waves," Phys. Rev. D43 (1991) 3907. [hep-th/0512188].

[6] P.-M. Zhang, M. Cariglia, M. Elbistan and P. A. Horvathy, "Scaling and conformal symmetries for plane gravitational waves," [arXiv:1905.08661 [gr-qc]].

[7] C. Duval, "Quelques procédures géométriques en dynamique des particules," Doctorat d'Etat ès Sciences (Aix-Marseille-II), 1982 (unpublished). See also G. Burdet, C. Duval and M. Perrin, "Cartan Structures On Galilean Manifolds: The chrono-projective Geometry," J. Math. Phys. 24 (1983) 1752. doi:10.1063/1.525927.

[8] M. Perrin, G. Burdet and C. Duval, "chrono-projective Invariance of the Five-dimensional Schrödinger Formalism," Class. Quant. Grav. 3 (1986) 461. doi:10.1088/0264-9381/3/3/020

[9] M. Cariglia, "Null lifts and projective dynamics," Annals Phys. 362 (2015) 642 doi:10.1016/j.aop.2015.09.002 [arXiv:1506.00714 [math-ph]].

[10] C. G. J. Jacobi, "Vorlesungen über Dynamik." Univ. Königsberg 1842-43. Herausg. A. Clebsch. Vierte Vorlesung: Das Princip der Erhaltung der lebendigen Kraft. Zweite ausg. C. G. J. Jacobi's Gesammelte Werke. Supplementband. Herausg. E. Lottner. Berlin Reimer (1884).

[11] R. Jackiw, "Introducing scaling symmetry," Phys. Today, 25 (1972) 23; U. Niederer, "The maximal kinematical symmetry group of the free Schrödinger equation," Helv. Phys. Acta 45 (1972) 802 C. R. Hagen, "Scale and conformal transformations in Galilean-covariant field theory," Phys. Rev. D5 (1972) 377.

[12] V. de Alfaro, S. Fubini and G. Furlan, "Conformal Invariance in Quantum Mechanics," Nuovo 
Cim. A 34 (1976) 569. doi:10.1007/BF02785666

[13] M. Henkel, "Local Scale Invariance and Strongly Anisotropic Equilibrium Critical Systems," Phys Rev. Lett. 78 (1997), 1940 ; "Phenomenology of local scale invariance: from conformal invariance to dynamical scaling," Nucl. Phys. B 641 (2002) 405

[14] C. Duval and P. A. Horvathy, "Non-relativistic conformal symmetries and NewtonCartan structures," J. Phys. A 42 (2009) 465206 doi:10.1088/1751-8113/42/46/465206 [arXiv:0904.0531 [math-ph]]

[15] G. S. Hall and J. D. Steele, "Conformal vector fields in general relativity," J. Math. Phys. 32 (1991) 1847. doi:10.1063/1.529249 ; G. S. Hall, "Symmetries and Curvature Structure in General Relativity", World Scientific (2004).

[16] M. W. Brinkmann, "Einstein spaces which are mapped conformally on each other," Math. Ann. 94 (1925) 119-145.

[17] M. Brdička, "On Gravitational Waves," Proc. Roy. Irish Acad. 54A (1951) 137.

[18] C. G. Torre, "Gravitational waves: Just plane symmetry," Gen. Rel. Grav. 38 (2006) 653 doi:10.1007/s10714-006-0255-8 [gr-qc/9907089].

[19] K. Andrzejewski and S. Prencel, "Memory effect, conformal symmetry and gravitational plane waves," Phys. Lett. B 782 (2018) 421 doi:10.1016/j.physletb.2018.05.072 [arXiv:1804.10979 $[\mathrm{gr}-\mathrm{qc}]]$.

[20] C. Duval, G. W. Gibbons, P. A. Horvathy and P.-M. Zhang, "Carroll symmetry of plane gravitational waves," Class. Quant. Grav. 34 (2017). doi.org/10.1088/1361-6382/aa7f62. [arXiv:1702.08284 [gr-qc]].

[21] P. M. Zhang, C. Duval, G. W. Gibbons and P. A. Horvathy, "Velocity Memory Effect for Polarized Gravitational Waves,” JCAP 1805 (2018) no.05, 030 doi:10.1088/1475-7516/2018/05/030 [arXiv:1802.09061 [gr-qc]].

[22] A. Ilderton, "Screw-symmetric gravitational waves: a double copy of the vortex," Phys. Lett. B 782 (2018) 22 doi:10.1016/j.physletb.2018.04.069 [arXiv:1804.07290 [gr-qc]].

[23] N. Dimakis, P. A. Terzis and T. Christodoulakis, "Integrability of geodesic motions in curved manifolds through non-local conserved charges," Phys. Rev. D 99 (2019) no.10, 104061 doi:10.1103/PhysRevD.99.104061 [arXiv:1901.07187 [gr-qc]].

[24] N.G. Van Kampen, "Transformation Groups and the Virial Theorem," Reports on Math. Physics 3, 235 (1972). 
[25] B. Nachtergaele and A. Verbeure, "Groups of canonical transformations and the virial-Noether theorem," Journal of Geometry and Physics, 3, 315-325 (1986)

[26] N. Ogawa, "A Note on the scale symmetry and Noether current," hep-th/9807086.

[27] T. Igata, "Scale invariance and constants of motion," PTEP 2018 (2018) no.6, 063E01 doi:10.1093/ptep/pty060 [arXiv:1804.03369 [hep-th]]. 